\section{RevistadePolítica Económica y Desarrollo Sostenible}

Centro Internacional de Política Económica para el Desarrollo Sostenible
Revista de Política Económica y Desarrollo Sostenible

EISSN: 2215-4167 • Vol. 7 (1) • Julio-Diciembre, 2021: 1-19

DOI: https://doi.org/10.15359/peds.7-1.2

URL: http://www.revistas.una.ac.cr/politicaeconomica

Revista electrónica semestral publicada por el Centro Internacional de Política Económica para el Desarrollo Sostenible

Universidad Nacional, Lagunilla, Heredia Apartado 2393-3000

\title{
Retos de la regulación del transporte remunerado de personas por medio de las plataformas digitales en Costa Rica
}

\author{
Challenges of the regulation of paid transportation of people through digital \\ platforms in Costa Rica
}

Yessica Espinoza González ${ }^{1}$

https://orcid.org/0000-0002-1394-9472

Sebastián Matarrita Lorente ${ }^{2}$

(iD) https://orcid.org/0000-0002-0322-8436

Fecha de recibido: 15 de febrero Fecha de corrección: 14 de mayo Fecha de aceptación: 30 de julio

\begin{abstract}
Resumen
El objetivo de este artículo es analizar los distintos aspectos que son importantes de regular en las plataformas digitales de transporte remunerado de personas en Costa Rica, con el fin de garantizar un equilibrio entre taxis autorizados, consumidores, las plataformas tecnológicas y los trabajadores de estas plataformas. La investigación es descriptiva con enfoque cualitativo. El principal hallazgo de la investigación es que, a pesar de que desde hace más de un quinquenio se introdujeron las plataformas digitales en el país, los avances en temas de regulación de calidad, precios y condiciones laborales son nulos, y muchos los retos que resolver. Conciliación entre los grupos de presión, modernización de las tarifas actuales del sector de taxis autorizados, regular aspectos de calidad son algunas de las recomendaciones para subsanar la situación actual.
\end{abstract}

Palabras clave: innovación, tecnológica, transporte público, regulación, mercado laboral.

\begin{abstract}
The goal of this essay is to analyze the most important aspects of digital-transportation-platforms regulation in Costa Rica, as the sector needs to balance the participation of official taxis, consumers, platforms and drivers from those platforms. This work is descriptive, and it was done under a qualitative basis. The main finding was that, even though those platforms were introduced more than 5 years ago, remaining work is left in terms of quality, pricing

1 Funcionaria de la Autoridad Regulatoria de los Servicios Públicos (Aresep), San José, Costa Rica. Es Bachiller en Economía por la Escuela de Economía de la Universidad Nacional, Heredia, Costa Rica. Estudiante de posgrado en la Maestría en Política Económica con énfasis en Economía de la Innovación, Cinpe- UNA, Heredia, Costa Rica, yessica.espinoza.gonzalez@est.una.ac.cr

2 Actualmente es Estudiante de posgrado en la Maestría en Política Económica con énfasis en Economía de la Innovación, Cinpe- UNA, Heredia, Costa Rica, sebasmatarrita90@hotmail.com
\end{abstract}


Revista de Política Económica y Desarrollo Sostenible • EISSN: 2215-4167

Vol. 7 (1) • Julio-Diciembre, 2021: 1-19

Espinoza González y

DOI: https://doi.org/10.15359/peds.7-1.2

Matarita Lorente

URL: http://www.revistas.una.ac.cr/politicaeconomica

and labor conditions. Conciliation between key groups, an upgrade in the pricing system from official taxis and quality adjustments in the sector are part of the recommendations aiming to solve the present issues.

Keywords: innovation, technology, public transport, regulation, labor market

\section{Introducción}

Desde la llegada de internet, la sociedad se ha involucrado en el desarrollo exponencial de no solamente nuevas tecnologías en diferentes ámbitos (como las telecomunicaciones, la educación, el transporte o el entretenimiento), sino que además ha impulsado necesidades en la población que cada vez buscan, con mayor exigencia, ser atendidas.

De esta manera, se ha llegado a lo que hoy se conoce como economía digital (Lian et al., 2021). Un concepto que se encuentra en pleno auge y que se basa en proporcionar de manera eficiente nuevos bienes y servicios al alcance de un clic. No cabe duda de las muchas ventajas y desafíos que la era digital ofrece a la sociedad.

Una de estas nuevas tecnologías, que ha llegado para revolucionar no solo la vida de las personas sino también los marcos regulatorios de los países, son las plataformas tecnológicas de transporte remunerado de pasajeros, como Uber, Cabify, Nova, DiDi, entre otras.

Estas plataformas tecnológicas conectan dos mercados: usuarios y conductores. Por un lado, están los usuarios que reciben un servicio fijando el punto de partida y de destino, con una tarifa prefijada, con acceso a través del móvil y una forma sencilla de pago. Por otro lado, está el mercado de conductores, para quienes estas plataformas presentan un modelo nuevo de trabajo derivado de la innovación tecnológica y la facilidad con la que se cuenta ahora para transmitir información. Según Lian et al. (2021), se crean relaciones laborales distintas pues el empleo tiene cierto carácter de independencia y la responsabilidad de los servicios recae enteramente en los usuarios de la aplicación.

Desde el 2015 en Costa Rica se implementó el servicio de transporte de Uber (referencia), y desde entonces ha sido el más popular en este grupo de plataformas junto con su homóloga china, DiDi. Dichas plataformas han ido adquiriendo un papel importante tanto dentro de la población, que goza de sus beneficios, como para su "competencia": el sector taxis, quienes critican la competencia desleal con la que tienen que tratar, al ser un transporte ilegal.

Sin embargo, es importante recalcar que, a pesar de que dicho servicio carece de un marco regulatorio, la cantidad de personas que lo utilizan como un ingreso secundario e incluso primario ha crecido en estos años, correspondido por una mayor cantidad de usuarios dispuestos a utilizar las plataformas.

Asimismo, los colaboradores de estas plataformas no cuentan con los mismos beneficios de que dispone un trabajo formal o uno dependiente. El seguro social debe ser adquirido por el colaborador y, dependiendo de la plataforma, no puede trabajar todas las horas que desee. De esta manera se cuestiona la calidad de estos empleos que, a pesar de todo, han venido creciendo. 
Revista de Política Económica y Desarrollo Sostenible • EISSN: 2215-4167

Vol. 7 (1) • Julio-Diciembre, 2021: 1-19

Espinoza González y

DOI: https://doi.org/10.15359/peds.7-1.2

Matarrita Lorente

URL: http://www.revistas.una.ac.cr/politicaeconomica

En Costa Rica se gestiona una ley relacionada con este servicio pero, a la fecha, el país no ha tomado una decisión; por tanto, hay una deuda en este sentido, pues a pesar de que el consumidor goza de los beneficios de las plataformas, hay muchas necesidades en materia de transporte que no han logrado ser atendidas. Tanto para el sector taxis como para las mismas plataformas es fundamental el debate de esta iniciativa.

De esta forma, se cuestiona: ¿Qué actores de la administración pública influyen en el marco regulatorio de estas plataformas? ¿Qué estrategias se han aplicado desde que ingresó la primera plataforma digital (Uber)? ¿Cuáles son los grupos de presión que participan en la regulación de estas plataformas? ¿En qué condiciones operan sus relaciones laborales?

Estas plataformas han formado una actividad económica difícil de estimar y con irregularidades legales en su operación. Por lo tanto, el objetivo del presente trabajo es identificar los retos en regulación que enfrentan las plataformas digitales de transporte remunerado de personas en Costa Rica.

El documento hace una revisión conceptual sobre las economías colaborativas, específicamente de plataformas digitales en el apartado 1 . En el apartado 2 se revisan los procedimientos metodológicos para la realización de este trabajo. En la sección 3 se discute el análisis sobre estas economías colaborativas en Costa Rica repasando los aspectos generales, los agentes de la actividad y los principales retos de regulación que enfrentan a nivel nacional. Finalmente, se abordan las conclusiones.

\section{Marco teórico-conceptual}

El uso de medios de transporte es una actividad común e indispensable en la sociedad moderna. Se emplean para movilizarse al trabajo, lugar de estudio, a algún lugar de entretenimiento o incluso para trasladar mercancías.

En específico, para describir un fenómeno como lo es el transporte remunerado de personas a través de plataformas digitales es necesario abordar ciertos conceptos sobre la digitalización de la economía y la innovación.

Sobre la naturaleza de la digitalización, Cruz (2017) considera que existe una fuerza sinérgica entre la globalización de las economías y la digitalización, como resultado de un avance tecnológico intrínseco en la sociedad. Por tanto, ambas -globalización y digitalización- se retroalimentan entre sí.

Como explica Carlota Pérez (2010), la introducción de una nueva tecnología conlleva cambios no solo en el consumo y modo de vida de las personas y la sociedad, sino también requiere una renovación en el aparato productivo existente, cambios institucionales y en las regulaciones de los países.

Con una tesis similar, donde el crecimiento económico y la digitalización de las economías se retroalimentan entre sí, Katz y Callorda (2015) consideran que los arreglos institucionales son necesarios para que estos se den, y concluyen que la digitalización tiene su impacto en la economía en tres áreas: crecimiento económico, posibilidad de nuevos emprendimientos y estímulo a la innovación. 
Revista de Política Económica y Desarrollo Sostenible • EISSN: 2215-4167

Vol. 7 (1) • Julio-Diciembre, 2021: 1-19

Espinoza González y

DOI: https://doi.org/10.15359/peds.7-1.2

Matarrita Lorente

URL: http://www.revistas.una.ac.cr/politicaeconomica

En este sentido, hay que recordar que la regulación y la innovación se influyen mutuamente: por un lado, la regulación afecta directamente al proceso innovador, mientras que, por otro, la innovación y el cambio tecnológico tienen un impacto significativo sobre la regulación. Entonces surge el dilema: ¿la innovación debe ser regulada o debe existir una regulación para la innovación?

Se entiende por "regulación” el conjunto de políticas de las que se vale el Gobierno para supervisar la actividad del mercado y el comportamiento de los actores en la economía. Esta intervención suele justificarse por la necesidad de asegurar el bienestar social cuando existen fallos de mercado que lo podrían impedir (Cotec, 2000).

La intervención gubernamental puede tomar distintos matices. En el caso particular de las plataformas tecnológicas en el sector transporte, se puede intervenir a través de los siguientes instrumentos regulatorios:

- Regulación de precios: Fijación de tarifas o precios por medio de modelos tarifarios (servicio al costo, precio tope, empresa modelo, entre otros), los cuales se puede implementar por:

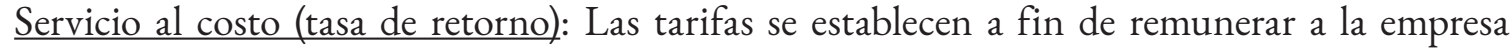
prestadora por los costos económicos en que incurre, sin poder recibir mayores ingresos. Este esquema de regulación se basa en establecer una tasa de retorno sobre el capital. La empresa regulada recibe los costos de operar el servicio más la tasa de retorno.

Tope de precios (price cap): El principal incentivo de la empresa para reducir sus costos es que, en este régimen, le es permitido retener toda la diferencia entre dichos costos y el precio tope. Promueve la eficiencia, pero ha sido criticado por llevar a las empresas a ofrecer servicio de una calidad inferior.

Empresa eficiente: Se toma como modelo una empresa que permite simular los costos que enfrenta una red operando y prestando los servicios en un determinado mercado y bajo las condiciones y características propias del país.

- Regulación de la calidad y condiciones del servicio: Imposición de determinados estándares de calidad y prestación del servicio.

La calidad de servicio de transporte público está constituida por criterios que reflejan el punto de vista del usuario sobre el servicio prestado, por ejemplo: servicio ofertado, accesibilidad, información, tiempo, atención al cliente, seguridad, comodidad, o impacto medioambiental.

- Regulación de entrada al mercado: Fijación del número de operadores o establecimientos de derechos exclusivos o de condiciones para la prestación del servicio.

- Sistemas de libre entrada, pero con aprobación o evaluación previa: Se dan licencias o títulos habilitantes.

- Regulación del mercado de trabajo: Se define el estatus legal de los trabajadores y se determina con qué derechos laborales cuentan y quiénes tienen la responsabilidad de velar por el cumplimiento de estos derechos. 
Revista de Política Económica y Desarrollo Sostenible • EISSN: 2215-4167

Vol. 7 (1) • Julio-Diciembre, 2021: 1-19

Espinoza González y

DOI: https://doi.org/10.15359/peds.7-1.2

Matarrita Lorente

URL: http://www.revistas.una.ac.cr/politicaeconomica

Tendiente a hacer esta definición, es importante tomar en cuenta los criterios de dependencia y autonomía de los conductores de estas plataformas. Dichos criterios son revisados por la CEPAL-OIT (2019) y aplican para todos los trabajadores que realizan actividades mediante plataformas digitales. En el cuadro 1 se resumen.

Cuadro 1. Criterios de dependencia y autonomía de los jornaleros digitales

\begin{tabular}{ll}
\hline Criterio & Trabajo de los jornaleros digitales mediante plataformas \\
\hline Tiempo de trabajo & $\begin{array}{l}\text { En principio, el trabajador puede organizar libremente su jornada, pero en varias } \\
\text { plataformas existen sistemas de incentivos que favorecen a los trabajadores con } \\
\text { mayor disponibilidad a la hora de asignar los pedidos. }\end{array}$ \\
Exclusividad del vínculo & $\begin{array}{l}\text { El trabajador puede desempeñarse a través de varias plataformas simultáneamente. } \\
\text { En muchos casos, el trabajo a través de la plataforma es una ocupación secundaria. }\end{array}$ \\
Bienes de capital & $\begin{array}{l}\text { El trabajador suele usar sus propios dispositivos tecnológicos (computadora, } \\
\text { teléfono celular) o medios de transporte (bicicleta, motocicleta, automóvil). }\end{array}$ \\
Definición del producto y precios & $\begin{array}{l}\text { El trabajador no tiene libertad para definir las características del producto ni su precio. } \\
\text { Supervisión }\end{array}$ \\
$\begin{array}{l}\text { En la mayoría de los casos, especialmente en el de las plataformas de intermediación } \\
\text { de tareas de ejecución local, existe una estrecha supervisión a través de las aplicaciones } \\
\text { de las plataformas. Se monitorea, por ejemplo, la ubicación del trabajador, la } \\
\text { duración de cada paso en la ejecución de su trabajo y la satisfacción del cliente. }\end{array}$ \\
$\begin{array}{l}\text { En las plataformas de intermediación laboral en la web, la intermediación consiste } \\
\text { principalmente en establecer el vínculo entre la oferta y la demanda. En muchas de } \\
\text { las plataformas que ofrecen oportunidades laborales de ejecución local, en cambio, } \\
\text { la intermediación incorpora tareas de supervisión y pago propias de un empleador. }\end{array}$ \\
\hline
\end{tabular}

Fuente: CEPAL-OIT (2019)

Considerando la regulación como instrumento para mejorar la eficiencia de los mercados, es importante tomar en cuenta, a su vez, que una de las deficiencias más importantes de los esquemas regulatorios es que no están pensados en términos de la innovación y, por lo general, están articulados a la estructura del pasado institucional y tecnológico del sector.

El informe de la OIT-CEPAL (2019) Coyuntura Laboral en América Latina y el Caribe. El futuro del trabajo en América Latina y el Caribe: antiguas y nuevas formas de empleo y los desafios para la regulación laboral sugiere considerar la soberanía del conductor sobre sus tiempos de trabajo, y comenta lo siguiente:

"Ampliar la soberanía sobre el tiempo. Los trabajadores necesitan una mayor autonomía sobre su tiempo de trabajo, sin dejar de satisfacer las necesidades de la empresa. Aprovechar la tecnología para ampliar las oportunidades y conciliar la vida profesional con la vida personal puede ayudarles a alcanzar este objetivo y encarar las presiones derivadas de la difuminación de 
la línea divisoria entre el tiempo de trabajo y el tiempo privado. Será preciso perseverar en los esfuerzos encaminados a aplicar límites máximos al tiempo de trabajo, además de medidas para mejorar la productividad" (OIT-CEPAL, 2019, p. 35)

Esto significa que la regulación no se formula en función de lo que no existe, sino que se diseña para regular lo que existe; es decir, la regulación de estos criterios de autonomía no es común en los esquemas regulatorios vigentes (Vargas, 2017).

Es por ello que el principal desafío del Estado consiste en hallar soluciones estables y viables en el futuro, que se integren a la economía de mercado y al mismo tiempo aseguren a todos los ciudadanos una protección colectiva suficiente.

En este respecto la CEPAL-OIT (2019) consideran dos recomendaciones sobre la representación colectiva de los conductores y el sistema de gobernanza internacional de las plataformas. Sobre la primera, CEPAL-OIT (2019, p. 20) recomiendan:

"Garantizar la representación colectiva de los trabajadores y los empleadores a través del diálogo social como bien público, promovido activamente a través de políticas públicas. Todos los trabajadores y todos los empleadores deberán disfrutar del reconocimiento de su libertad sindical y del derecho de negociación colectiva, con el Estado como garante de esos derechos".

Sobre las condiciones mínimas que deben tener los colaboradores de estas plataformas, consideran que:

"Debería establecerse un sistema de gobernanza internacional de las plataformas digitales del trabajo que exija a estas plataformas (y a sus clientes) que respeten determinados derechos y protecciones mínimas. Los avances tecnológicos requieren también de la reglamentación del uso de datos y de la responsabilidad sobre el control de los algoritmos en el mundo del trabajo”.

Todos estos elementos teóricos se acoplan al tema de estudio desarrollado en este trabajo, considerando la introducción de las plataformas tecnológicas en el sector transporte como una innovación radical que conlleva una transformación del patrón tecnológico y organizativo de las sociedades.

\section{Metodología e información}

Esta investigación tiene un enfoque cualitativo, pues se trata de un enfoque flexible y la recolección de información no involucra datos numéricos o procesos estadísticos. Asimismo, es de tipo descriptivo, dado que se realizará una caracterización del mercado de vehículos de alquiler con conductor y su entorno para establecer los principales retos que se presentan dentro del mismo. 
El objeto de estudio es el transporte remunerado de personas a través de plataformas digitales, y de este el interés está en analizar los retos de regulación vigentes en Costa Rica.

Para el logro del objetivo planteado se requiere de tres etapas o fases: A) definición, búsqueda y revisión de información, así como diseño de la investigación, B) recolección, preparación y análisis de datos, y C) síntesis de resultados, conclusiones y recomendaciones.

Por tanto, se acude a fuentes primarias y secundarias como recursos bibliográficos y estudios con resultados relacionados a esta investigación que generen información de primera mano de los actores involucrados, así como otros trabajos que hayan avanzado en el tema.

Como instrumentos, este trabajo sistematiza la información recopilada (documentos, registros, materiales): normativas actuales y propuestas de Ley en temas de regulación y transporte público a nivel institucional.

La muestra parte de las siguientes instituciones: Ministerio de Obras Públicas y Transportes (MOPT) a través del Consejo de Transporte Público (CTP), Autoridad Reguladora de los Servicios Públicos (ARESEP), Ministerio de Trabajo y Seguridad Social, Presidencia y Asamblea Legislativa.

\section{Resultados}

\subsection{Economía colaborativa del transporte remunerado de personas en Costa Rica}

\subsubsection{Caracterización del sector}

Un taxi es un modo de transporte que ofrece un servicio rápido y cómodo de puerta a puerta a los usuarios. Es un complemento a otros medios de transporte público, que tiene como objetivo ofrecer una mejor movilidad a los usuarios; en este sentido el servicio de transporte ofrecido por las plataformas tecnológicas se asemeja al servicio que brindan los taxis.

Sin embargo, a diferencia del servicio de transporte que brindan las plataformas tecnológicas, en Costa Rica los taxis son un servicio público, cuyo titular es el Estado y, por tanto, está supeditado a una serie de regulaciones por parte del Consejo de Transporte Público (CTP) y la Autoridad Reguladora de los Servicios Públicos (ARESEP), quienes a su vez, al ser instituciones estatales y por las características propias del servicio, están sujetos en algunos casos a decisiones que dependen de la Asamblea Legislativa y la Presidencia o Poder Ejecutivo.

El marco regulatorio del transporte público de pasajeros, específicamente en el sector taxi, data de 1999 y se rige a través del Consejo de Transporte Público (CTP) por la Ley N 7969: Ley Reguladora del Servicio Público de Transporte Remunerado de Personas en Vehículos en la Modalidad Taxi. Es mediante esta ley que la habilitación o el uso del servicio se ven antecedidos por el cumplimiento de ciertas obligaciones o condiciones para el ejercicio de la actividad. 
Revista de Política Económica y Desarrollo Sostenible • EISSN: 2215-4167

Vol. 7 (1) • Julio-Diciembre, 2021: 1-19

Espinoza González y

DOI: https://doi.org/10.15359/peds.7-1.2

Matarrita Lorente

URL: http://www.revistas.una.ac.cr/politicaeconomica

Entre algunas de estas condiciones se encuentran: concesión o permiso de operación, revisión técnica vehicular dos veces al año, contar con un taxímetro, pago de cánones del servicio público, pago de pólizas por lesión o muerte de terceros y daños a propiedad, horario mínimo de conducción ${ }^{3}$, entre otras.

Adicionalmente, y como parte de su condición de servicio público, el sector taxis también es regulado por la ARESEP, a través de la Ley $\mathrm{N}^{\circ}$ 7593: Ley de la Autoridad Reguladora de los Servicios Públicos. Esta entidad fija las tarifas que deben cobrar los taxis, con el régimen del servicio al costo (tarifa banderazo, variable, por espera y demora, para taxis base de operación regular y aeropuerto), siendo estas de carácter obligatorio para los prestadores del servicio.

En este sentido es que el gremio de taxistas ha manifestado en diversas ocasiones que el servicio de transporte ofrecido por los taxistas y el de las plataformas tecnológicas son dos modalidades que conviven en el mercado costarricense desde hace aproximadamente cinco ańos en condiciones desiguales.

A finales del 2019 (Vargas, 2019), el líder de la Unión de Taxistas Costarricenses (UTC), Rubén Vargas, declaró que:

"El taxista tiene que exigir un cambio, ahora vamos por otro lado. El taxista ya no va a aceptar ninguna regulación; lo que va hacer es exigir el cumplimiento de las leyes de este país, las leyes que protegen al taxista”.

A través de otro medio (CRHoy.com, 2019), el líder de la UTC justificó que:

"Ante el nacimiento de nuevos métodos de transporte, que no han sido regulados por su modernidad, Costa Rica, igual que todos los países del mundo, está en la necesidad de crear leyes que impongan parámetros de regulación a estas plataformas, ya que las mismas trabajan a la libre con derechos y obligaciones impuestos por ellas mismas, no por el Estado”.

El CTP a través del acuerdo DAJ-2015002929 del 10 de setiembre del 2015 emitió criterio en el que manifestó que el transporte privado remunerado de personas que se brinda por medio de la empresa Uber es ilegal, dado que no cuenta con las autorizaciones correspondientes; reafirmando que el titular único del servicio de transporte remunerado de personas es el Estado costarricense. No es posible brindar este servicio público si no se cuenta con autorización por parte del CTP.

Del criterio jurídico sobre Uber emitido el 25 de agosto del 2015 por el CTP, se desprende:

- La empresa Uber no se encuentra autorizada en ninguna modalidad de transporte remunerado de personas, y dado que toda prestación de transporte remunerado es de la titularidad del Estado, al no contarse con la autorización debida, provoca que el servicio se catalogue como ilegal, por corresponder a un servicio público.

- Ante las manifestaciones de algunos ante la opinión pública, sobre la pertinencia y viabilidad de operación de Uber amparadas al Tratado de Libre Comercio entre Estados

$3 \mathrm{Al}$ menos 12 horas diarias. 
Revista de Política Económica y Desarrollo Sostenible • EISSN: 2215-4167

Vol. 7 (1) • Julio-Diciembre, 2021: 1-19

Espinoza González y

DOI: https://doi.org/10.15359/peds.7-1.2

Matarrita Lorente

URL: http://www.revistas.una.ac.cr/politicaeconomica

Unidos y Costa Rica, es importante destacar que, siguiendo la jerarquía de normas, fuentes de Derecho y el principio de legalidad, ninguna convención, convenio o tratado suscrito entre Costa Rica y su homólogo puede contravenir el ordenamiento jurídico interno.

En esta misma línea, la posición de la ARESEP es que toda prestación remunerada de transporte en el país debe estar avalada por el CTP. Del criterio OF-1051-RG-2018 se desprende:

- De acuerdo con la Ley, para prestar servicio de transporte remunerado de personas en el país se debe contar con una concesión o permiso emitido por el Consejo de Transporte Público; de lo contrario, será una prestación ilegal o no autorizada, según lo ha resuelto la Sala Constitucional.

- ARESEP no pueden clausurar o cerrar la app porque es un servicio tercerizado; es decir, que la compañía Uber, al no ser la dueña de los vehículos que ofrecen el servicio de transporte, no puede ser sancionada.

- La Policía de Tránsito tiene la potestad de detener aquellos conductores que sean sorprendidos dando un servicio "pirata”, entre ellos Uber, y otros que utilicen plataformas tecnológicas para atraer clientes.

Con este antecedente jurídico, cualquier plataforma tecnológica de transporte remunerado de personas tendría que solicitar el visto bueno del CTP para ofrecer el servicio; sin embargo, desde que estas empresas llegaron al país en el 2015 no solicitaron permisos y se refugian en la figura legal de "comunidades de autoabastecimiento" para operar una aplicación que une a socios conductores con clientes para llevarlos de un sitio a otro en su automóvil. En este sentido, pretenden aprovecharse de un vacío legal, pues en Costa Rica no existe ninguna ley que regule la economía colaborativa.

Sobre las "comunidades de autoabastecimiento", es importante entender que se refiere a un término que ha utilizado la Procuradora General de la República para referirse al autoabastecimiento de servicios básicos como el agua y la electricidad, donde se permite el consumo de estos bienes en pequeñas comunidades sin la prestación de su servicio (Madrigal, 2015). Ante la afirmación de que las plataformas de transporte remunerado operan bajo esta figura, como lo hizo Uber en una carta abierta al Gobierno (Echandi, 2018), la procuradora consideró que dicho término no tiene la amplitud para contemplar las actividades de la plataforma (Madrigal, 2015).

De manera más amplia, las plataformas digitales operan en un modelo de economías colaborativas. Lizano (2018, p. 81) discute este concepto como "un nuevo modelo de intercambio económico que se basa en 3 principios: interacción entre productor y consumidor, que mantienen un diálogo continuo, conexión entre pares gracias a las tecnologías y especialmente digitales y la colaboración”. Respondiendo a esa descripción, los servicios de plataformas como Uber, DiDi o InDriver son usualmente enmarcadas como economías colaborativas.

Entre los avances que la Administración ha tenido con este tema, está la elaboración de un proyecto de ley (Ley $\mathrm{N}^{\circ}$ 21587) que pretende ordenar este servicio, que actualmente opera por la libre, y se rige solo por las reglas de oferta y demanda, sin regulaciones ni trámites burocráticos; sin embargo, es un tema de discusión y análisis aún en la Asamblea Legislativa. 
Revista de Política Económica y Desarrollo Sostenible • EISSN: 2215-4167

Vol. 7 (1) • Julio-Diciembre, 2021: 1-19

Espinoza González y

DOI: https://doi.org/10.15359/peds.7-1.2

Matarita Lorente

URL: http://www.revistas.una.ac.cr/politicaeconomica

A la fecha han pasado casi seis años desde que las plataformas tecnológicas funcionan en el país; el último dato revelado por la misma la empresa es que Uber cuenta con 29.000 conductores y brinda servicios de transporte aproximadamente a 970.000 clientes. Asimismo, invirtió cerca de $\$ 30$ millones en los últimos 4 años en su centro de operaciones de Latinoamérica, que opera desde Costa Rica (elfinanciero.com, 2019).

A pesar del crecimiento de la actividad, los avances en regulación parecen ser pocos; lo cierto es que Costa Rica está atrasada en la generación de regulaciones a través de plataformas digitales. Los marcos regulatorios que en el momento existen, aunque han sido funcionales en su momento, están obsoletos ante la implementación de innovaciones tecnológicas.

La posición de Uber es clara y sugiere que la actividad sea regulada por el resguardo de sus colaboradores. Mediante una carta a la Asamblea Legislativa, Madriz, A. (2020) expresó que:

- "Hoy, en medio de una de las situaciones más difíciles que ha vivido Costa Rica y el mundo, es urgente garantizar la seguridad jurídica de los miles de personas que dependen de los ingresos que se generan a través de aplicaciones como la que ofrecemos”.

De igual manera, el vocero de DiDi (Hidalgo, K., 22 de setiembre, 2020) hizo constar que:

- "Hay que aceptar la tecnología que llegó para quedarse y tiene un impacto muy positivo en cómo los ticos se mueven. Queremos que sea una regulación que promueva el uso de la tecnología, que sea de libre entrada para la diferente competencia y que priorice el centro con beneficios para los usuarios y socios conductores”.

Según se extrae de la Reforma al Sistema de Transporte Remunerado de Personas y Regulación de las Empresas de Plataformas Tecnológicas de Transporte, dictamen negativo unánime del 11 de setiembre del 2019 (pp. 43-44), el antepenúltimo proyecto de ley se rechazó por las siguientes razones

"Consideramos que tanto el texto base presentado por el Poder Ejecutivo, como los borradores de texto presentados por los taxistas, contienen una serie de artículos con posibles roces de constitucionalidad imposibles de subsanar, y otras inconsistencias cuyos posibles cambios afectarían la conexidad del mismo. (...) Finalmente consideramos que el proyecto de ley $\mathrm{N}^{\circ}$ 21.228 no contiene una visión moderna sobre la prestación de este servicio de transporte; por el contrario, pretende establecer regulaciones costosas en contra del uso de la tecnología en la prestación de servicios de transporte en general.

Debido a lo anterior, los diputados miembros de la Comisión Permanente de Asuntos Económicos consideran que la mejor solución para que los servicios de transporte por medio de plataformas tecnológicas y el sistema de transporte público remunerado de personas modalidad taxi puedan vivir con una competencia leal y respetando el ordenamiento jurídico costarricense no era aprobando el expediente $\mathrm{N}^{\circ} 21.228$, sino archivando este expediente y presentando a la corriente legislativa un nuevo proyecto de ley que respete una actividad de índole privada sin violentar los derechos que tienen los servicios de transporte público” 
No obstante, a pesar de que se planteó otro proyecto (el No. 21.587: Ley Reguladora de los Servicios de Transporte de Personas por Medio de Plataformas Tecnológicas) que subsana las debilidades del anterior, la oposición por parte de los taxistas a cualquier ley que regule el transporte brindado por plataformas y la presión ejercida sobre la administración de Carlos Alvarado llevaron a este último, mediante el decreto ejecutivo número 42501-MP, a desestimar sin mayor explicación la discusión del proyecto de ley pretendido.

\subsection{2 ¿Qué tipo de aspectos se deben regular?}

Cuando se habla del mercado de transporte remunerado de personas y las plataformas tecnológicas, es posible pensar en varios instrumentos que se pueden utilizar para regular el servicio.

Tal y como se explicó anteriormente, el servicio de taxis es el que más se asemeja al ofrecido por estas plataformas y es regulado en precios, capacidad, calidad y también por la parte del mercado laboral; por tanto, seguidamente se va a analizar cada uno de estos instrumentos considerando el contexto actual de innovación y la situación vigente.

\subsubsection{Regulación de precios, capacidad y calidad}

Con la irrupción de nuevos modelos de negocio basados en la tecnología y el ecosistema digital, el país requiere de la modernización de su marco regulatorio lo antes posible.

Actualmente los taxis rojos o anaranjados (autorizados) son regulados tanto en su capacidad y calidad, como en la tarifa, con el fin último de garantizar al usuario que los prestadores cumplan con un servicio confiable, continuo, oportuno y de prestación óptima, según lo establecido en el artículo 5 de la Ley $N^{\circ}$ 7593: Ley de la Autoridad Reguladora de los Servicios Públicos.

Si bien es necesario que las plataformas digitales ofrezcan productos que cumplan con todas estas normas, también es cierto que la diversificación que han llegado a ofrecer los servicios de transporte por medios digitales ha sido positivo para el consumidor, frente a un servicio de taxis que arrastra muchas deficiencias y con un marco regulatorio que limita la operación de los mismos.

Los principales aspectos que se regulan en Costa Rica para el servicio de taxi son:

\section{- Precio}

En Costa Rica la regulación de precios del servicio de taxis está a cargo de la ARESEP, quien regula tanto los taxis rojos (base regular) como los taxis anaranjados (base especial), a través de fijaciones ordinarias y extraordinarias, y de cuatro tarifas: tarifa banderazo, variable, por espera y por demora.

Las tarifas que fija la ARESEP se realizan a través de la estructura de costos del servicio y se toman en cuenta gastos fijos como: activos, salarios, gastos administrativos, retribución al concesionario (tasa de retorno), y las variables de combustible, reparación y mantenimiento, llantas y lubricantes, entre otros. 
La última fijación ordinaria realizada por la institución, que es la que contabiliza todas estas variables, data del 2015 y ya algunos valores están caducos; mientras que las fijaciones extraordinarias que contabilizan la inflación, el tipo de cambio y la variación de los salarios y cánones se realiza semestralmente sin falta.

Ante la presencia de un nuevo competidor, aunque sea ilegal, ARESEP debería replantear la modificación de su metodología tarifaria o al menos la realización de una actualización de datos a través de una nueva fijación ordinaria; aunque el precio no es el único factor que hace que un usuario utilice un servicio, las bajas tarifas que ofrecen las plataformas tecnológicas han sido factor clave para el traslado de muchos usuarios hacia el uso de las aplicaciones.

\section{- Capacidad}

Durante décadas, el sector del taxi ha vivido en régimen de oligopolio, fundamentado en las llamadas licencias que ha concedido la administración pública (Ministerio de Obras Públicas y Transportes), para regular de manera directa la oferta del sector.

La concesión de licencias implica fijar unos requisitos para determinar quién puede optar a ella. Esto no sólo limita la oferta, sino que se determinan indirectamente los costes mediante cargas fiscales y demás condiciones para ser apto.

Uber representa una molestia para los taxis regulares que han sufrido la disminución de ingresos debido al aumento de la competencia. La plataforma tecnológica no está regulada por las normas de concesión de licencias, a diferencia de los taxis regulares, lo que para algunos taxistas significa la convivencia diaria con la competencia desleal.

\section{- Calidad}

Usualmente la regulación en la calidad en el transporte público se rige por la adopción de normas de calidad según las mejores prácticas aplicables. En Costa Rica se homologaron y adaptaron las normas europeas de calidad del transporte público.

La primera norma aprobada es la INTE G20:2020 Transporte público de personas. Definición de la calidad de servicio, objetivo y mediciones, la cual describe los criterios de calidad aplicables (servicio ofertado, accesibilidad, información, tiempo, atención al usuario, comodidad, seguridad e impacto medioambiental) y los mecanismos de evaluación.

La segunda norma aprobada es la INTE G21:2020 Transporte público de pasajeros. Requisitos básicos y recomendaciones para los sistemas de medición de la calidad de servicio producida, que describe las buenas prácticas dirigidas a los prestadores para una correcta implementación de la calidad de servicio. 
Revista de Política Económica y Desarrollo Sostenible • EISSN: 2215-4167

Vol. 7 (1) • Julio-Diciembre, 2021: 1-19

Espinoza González y

DOI: https://doi.org/10.15359/peds.7-1.2

Matarrita Lorente

URL: http://www.revistas.una.ac.cr/politicaeconomica

Estas normas son de carácter voluntario y se empezaron a implementar a partir del 2020 por parte de la ARESEP como un plan piloto con los prestadores. La idea es que de manera paulatina y ordenada la norma pueda ser de carácter obligatorio.

Empresas y/o asociaciones de taxi como Cootaxa R.L, Taxis Unidos Desamparadeños y CoopeIrazú son de las primeras en querer adoptar estas normas de forma voluntaria; sin embargo, hay aún un gran desafío para que los usuarios recuperen la confianza en los taxis rojos, frente a un servicio ilegal que hasta la fecha ha suministrado a los usuarios una buena atención, viajes cómodos con vehículos limpios, accesibilidad de puerta a puerta, entre otras ventajas comparativas.

Debido a lo anterior es que, con la regulación de las plataformas digitales, se pretende reformar y mejorar la normativa relacionada con el servicio público de transporte remunerado de personas modalidad taxi, con el fin de garantizar una mayor competitividad, mejorar la calidad del servicio que se brinda y establecer un escenario de armonía y competencia sana entre los diferentes oferentes de transporte de personas (proyecto de ley 21.587: Ley Reguladora de los Servicios de Transporte de Personas por Medio de Plataformas Tecnológicas; actualmente este proyecto no se ha vuelto a convocar).

En este sentido, el mayor reto para el país es ponerse de acuerdo para implementar de manera rápida y efectiva una normativa que modernice el servicio existente e incluya la nueva oferta de transporte que actualmente es ilegal, para no perjudicar a los consumidores finales, los taxis autorizados y el servicio de transporte of recido por las plataformas digitales.

Hasta la fecha, la Fuerza Roja (taxis autorizados) es la que más ha luchado para que se regulen las plataformas tecnológicas de transporte; asimismo la empresa Uber y DiDi han manifestado su interés en dialogar sobre la ley que podría regularlas; sin embargo, está en manos del Gobierno la discusión del proyecto.

El Ejecutivo tiene el control de la agenda de los proyectos que se discuten en el Congreso, convocando y desconvocando las propuestas legislativas que desea se discutan en el Plenario Legislativo; por tanto, por el momento es el Ejecutivo quien retrasa la regulación de las plataformas digitales (solo en el periodo enero-agosto del año 2021). Al final esta normativa debe ser el resultado de la coordinación de acciones entre todas las partes, con el Gobierno como mediador para lograr un acuerdo conciliatorio.

\section{- Regulación de las Condiciones Laborales}

La innovación tecnológica ha llevado a digitalizar las relaciones laborales, de forma que los actores involucrados en una actividad lucrativa establezcan una relación mínima entre el empleador y el empleado; no obstante, siempre conectada a través de una plataforma digital.

Hay desafíos en que la simple realización de la actividad y la rendición de cuentas sea monitoreada exclusivamente por una plataforma digital privada. Una de las principales es asegurar la calidad de las personas que trabajan en estos medios. 
Revista de Política Económica y Desarrollo Sostenible • EISSN: 2215-4167

Vol. 7 (1) • Julio-Diciembre, 2021: 1-19

Espinoza González y

DOI: https://doi.org/10.15359/peds.7-1.2

Matarrita Lorente

URL: http://www.revistas.una.ac.cr/politicaeconomica

Para el 2021, las plataformas digitales representan un nuevo reto en la innovación de los contratos laborales. Los trabajadores de dichas plataformas han planteado nuevas características en los siguientes términos: el tiempo de trabajo es flexible, pero con incentivos; no hay exclusividad del vínculo: se usan varias plataformas (conductor de InDriver y Uber simultáneamente), los bienes de capital no pertenecen a la plataforma, los precios son determinados por la plataforma, la supervisión se realiza vía sistema de comentarios y valoración, y hay una intermediación mínima entre la plataforma y el colaborador (CEPAL citado en OIT, 2019, p. 35)

Estas características implican cierto desapego y autonomía entre la plataforma y el colaborador. Asimismo, el carácter privado de la operación implica que el mismo colaborador queda desprotegido en aspectos de seguridad social, dado su estado incierto como trabajador.

En este aspecto se han considerado los siguientes elementos para regular este mercado: I. Ampliar la soberanía sobre el tiempo II. Perseverar los esfuerzos encaminados a aplicar límites máximos al tiempo trabajado, además de medidas para mejorar la productividad. III. Establecer un sistema de gobernanza internacional de las plataformas digitales del trabajo que exija a estas plataformas respetar determinados derechos y protecciones mínimas (OIT, 2019)

En el caso de Costa Rica, la perfilación laboral de estos colaboradores es todavía incierta; es decir, quienes generen un ingreso, primario o secundario, a partir de estas plataformas, no son considerados ni como dependientes ni como independientes. Su caracterización privatiza los contratos laborales y la seguridad social de los colaboradores queda a cuenta propia.

A nivel institucional se ha propuesto la Ley 21.587, Ley Reguladora de los Servicios de Transporte de Personas por Medio de Plataformas Tecnológicas. Cuenta con el apoyo y promoción de Uber y DiDi (ameliarueda.com, 2020), ya que propone regular estas actividades y dar cobertura en seguridad social a todos los trabajadores; sin embargo, se encuentra todavía en discusión, como se indicó anteriormente. Las propuestas del proyecto se resumen en el anexo 1.

Uno de los obstáculos para la aprobación de esta Ley es la presión que ejercen los taxistas rojos como transportistas públicos. Consideran que la actividad es competencia directa para el negocio de los taxis y este, según los taxistas, ha venido en decadencia desde que ingresó Uber hace más de 5 años, dado que las tarifas del taxi están sujetas al control del Estado, mientras las plataformas regulan libremente sus propias tarifas.

El proyecto ya fue desestimado una vez, dado que no obedece a los intereses nacionales que ocupan la agenda política al día de hoy. Asimismo, a las partes involucradas tampoco les urge su aprobación: las plataformas todavía estudian alternativas de regulación, los taxistas siguen presionando para que no se regule la actividad con las condiciones actuales, y el Estado parece estar más preocupado por los contratos monetarios que puede generar la formalización de estas entidades, como las licencias de inscripción propuestas por el MOPT, que por la seguridad social de sus colaboradores. 
Revista de Política Económica y Desarrollo Sostenible • EISSN: 2215-4167

Vol. 7 (1) • Julio-Diciembre, 2021: 1-19

Espinoza González y

DOI: https://doi.org/10.15359/peds.7-1.2

Matarrita Lorente

URL: http://www.revistas.una.ac.cr/politicaeconomica

\section{Conclusiones y recomendaciones}

La irrupción creciente de las plataformas digitales aventura cambios importantes en el modo de organización y funcionamiento de las economías. Esta circunstancia puede aportar importantes beneficios, pero, al mismo tiempo, ocasiona desafíos nuevos para las autoridades económicas.

Desde su llegada en 2015, a través de Uber, las plataformas de transporte remunerado de personas demuestran los retos que existen en las instituciones de Costa Rica para regular algunas economías colaborativas. Uno de los aspectos más importantes a valorar es la competencia del sector y el estado legal en la operación de estas plataformas. El estado legal de las economías colaborativas como comunidades de autoabastecimiento, en las cuales Uber quiere incluirse, genera fricciones entre las empresas privadas y algunos gremios del transporte público; asimismo, la seguridad social de los conductores que prestan su servicio tiene aspectos de carga laboral que deben ser regulados por el Estado.

Ante esta problemática, agentes como el MOPT, ARESEP y la Asamblea Legislativa, junto con las empresas privadas como Uber y DiDi, han propuesto mecanismos de regulación a través de proyectos de ley; sin embargo, para mayo del 2021 ninguno de tales proyectos ha sido aprobado y, en razón de que el último proyecto ni siquiera se ha convocado debido a la presión ejercida por el sector taxis al Gobierno, no se ha siquiera determinado si el actual proyecto subsana las debilidades del anterior.

A pesar de que la situación jurídica no se resuelve, la actividad no cesa y, por el contrario, demuestra crecimiento. Esto sugiere que hay relaciones laborales que nacen y se forman a partir de condiciones establecidas por entes privados. Por tanto, estas condiciones laborales siguen pendientes como objeto de análisis en la regulación de este servicio, siendo clave la protección social necesaria para llevar a cabo la actividad.

El análisis realizado le supone al Gobierno de Costa Rica los siguientes retos, en un contexto de adaptación de la regulación vigente a las condiciones actuales en regulación:

a. Conciliación con los grupos de presión: Los taxistas, mediante huelgas, bloqueos y manifestaciones, han saboteado cualquier esfuerzo formal por regular el transporte remunerado de personas a través de plataformas. La reacción positiva del Gobierno ante sus demandas demuestra el estatus de este grupo en el transporte público y el poder de decisión que tiene sobre los intentos de regulación. Su colaboración en cualquier esfuerzo de regulación es imperante para lograr llevarlo a cabo.

b. Modernización de la regulación de tarifas de taxis existentes: Las ventajas tecnológicas que han demostrado las plataformas digitales sirven como ejemplo de la eficiencia en los medios de pago electrónicos, dada la aceptación que ha tenido por parte de los consumidores. En Costa Rica, el uso de aplicaciones digitales e instrumentos de pago electrónicos como plataformas digitales análogas para los taxis y tarjetas para pago sin contacto de membresía mensual o anual para el próximo tren eléctrico y la red de buses, son alternativas que se sugieren implementar en el transporte público. 
Revista de Política Económica y Desarrollo Sostenible • EISSN: 2215-4167

Vol. 7 (1) • Julio-Diciembre, 2021: 1-19

Espinoza González y

DOI: https://doi.org/10.15359/peds.7-1.2

Matarrita Lorente

URL: http://www.revistas.una.ac.cr/politicaeconomica

c. Regular aspectos de calidad y operación del servicio ofrecido por las plataformas tecnológicas: Deben establecerse ciertos derechos de propiedad para asegurar la calidad y la seguridad en los servicios proveídos en estas economías colaborativas. Aspectos técnicos sobre el vehículo, listados como requisitos mínimos y pertinentes con la prestación de servicio de transporte deben estandarizarse para dichas plataformas tecnológicas. Hay que capacitar previamente a los conductores en la prestación del servicio; la certificación de capacidades, en colaboración con instituciones de aprendizaje como el INA, se debe establecer y estandarizar al igual que las especificaciones técnicas para aquellas plataformas que transporten personas.

d. Asegurar las condiciones dignas de los colaboradores: Si bien dichas aplicaciones ofrecen una ocupación con horario flexible, se debe velar por los máximos de horas trabajadas semanalmente admitidos por Ley. Asimismo, las prestaciones legales que se negocian como los contratos por hora y jornada entre el conductor y la plataforma deben ser reguladas por el Estado, para asegurar cargas máximas y evitar situaciones de explotación o de inflexibilidad en las horas previamente establecidas.

En este sentido, se insta a implementar una regulación con una visión moderna, que no sea costosa, que incentive la calidad, que tome en cuenta el punto de vista de los usuarios, de los trabajadores de las plataformas tecnológicas y también de los trabajadores del servicio público autorizado.

Es impostergable trabajar en estos aspectos para poder satisfacer las necesidades tanto de los gremios en el transporte público como del nuevo servicio de transporte ofrecido mediante las plataformas tecnológicas y sus empleados, así como de los consumidores.

\section{Referencias}

Asamblea Legislativa de la República de Costa Rica. Proyecto de Ley Reguladora de los Servicios de Transporte de Personas por Medio de Plataformas Tecnológicas. https://semanariouniversidad. com/wp-content/uploads/2019/09/Texto-Plataformas-de-transporte-v-FINAL.pdf

Asamblea Legislativa de la República de Costa Rica (1965). Ley Reguladora del Transporte Remunerado de Personas en Vebiculos Automotores № 3503.

Asamblea Legislativa de la República de Costa Rica (1996). Ley de la Autoridad Reguladora de los Servicios Públicos $N^{\circ}$ 7593. http://www.pgrweb.go.cr/scij/Busqueda/Normativa/Normas/nrm texto completo.aspx? param $1=$ NRTC\&nValor $1=1 \&$ nValor $2=26314 \& \mathrm{nValor} 3=125008 \& \mathrm{pa}-$ $\underline{\operatorname{ram} 2=1 \& s t r T i p M=T C \& 1 R e s u l t a d o=4 \& s t r S i m=s i m p}$

Asamblea Legislativa de la República de Costa Rica (11 de setiembre de 2019). Reforma al Sistema de Transporte Remunerado de Personas y Regulación de las Empresas de Plataformas Tecnológicas de Transporte. Expediente N²1.228. http://www.asamblea.go.cr/sd/SiteAssets/ Lists/Consultas\%20Biblioteca/EditForm/dictamen\%20negativo\%20proyecto\%2021228.pdf 
Revista de Política Económica y Desarrollo Sostenible • EISSN: 2215-4167

Vol. 7 (1) • Julio-Diciembre, 2021: 1-19

Espinoza González y

DOI: https://doi.org/10.15359/peds.7-1.2

Matarrita Lorente

URL: http://www.revistas.una.ac.cr/politicaeconomica

Avendaño, M. (09 de enero del 2020). Uber revela nuevos datos. El Financiero. https://www. elfinancierocr.com/economia-y-politica/uber-revela-nuevos-datos-tiene-28000-conductores/ J6S7DK37QJFXFMCZTABBMO65Z4/story/

Barrantes, R. (2018). Teoría de la regulación. http://files.pucp.edu.pe/departamento/economia/ME004.pdf

Busquets, J. (13 de febrero del 2019). Las plataformas de transporte: globalidad y costes de la innovación. https://www.expansion.com/blogs/sociedad-empresa-digital/2019/02/13/lasplataformas-de-transporte-en-las.html

Campos Rojas, A. (09 de diciembre del 2019). Tecnología vs Legislación: Las plataformas colaborativas y la implicación de las leyes actuales. La República. https:/www.larepublica.net/noticia/ tecnologia-vs-legislacion-las-plataformas-colaborativas-y-la-inaplicabilidad-de-las-leyes-actuales

CEPAL-OIT (2019). Coyuntura laboral en América Latina y el Caribe. El futuro del trabajo en América Latina y el Caribe: antiguas y nuevas formas de empleo y los desafios para la regulación laboral. Santiago de Chile: Naciones Unidas.

Comisión para Promover la Competencia (2018). Regulación y competencia en el transporte remunerado de personas: Modalidad taxi y similares. https://www.coprocom.go.cr/publicaciones/estudios mercado/ESTUDIO\%20DE\%20MERCADO\%20DE\%20TAXIS\%20Y\%20SIMILARES.pdf

Corona, S. (16 de julio del 2015). El DF es la primera ciudad que regula Uber en América Latina. El Pais. https://elpais.com/internacional/2015/07/16/actualidad/1437073257_032569.html

COTEC (2014). Impacto de la regulación sobre la innovación. http://informecotec.es/media/J21 Impac_Regu_Innov.pdf

CRHoy (2019). Taxistas se manifestarán nuevamente el martes contra Uber. CRhoy.com. https:// www.crhoy.com/nacionales/taxistas-se-manifestaran-el-martes-en-san-jose/

Cruz, J. (2017). Las transformaciones de las relaciones laborales ante la digitalización de la economía. Temas laborales, núm. 138/2017, págs. 13-47. Universidad de Sevilla. https://dialnet.unirioja.es/ servlet/articulo?codigo $=6552388$

Echandi, A. (6 de septiembre del 2018). Uber Costa Rica envía carta abierta al gobierno. Uber.com. https://www.uber.com/es-CR/newsroom/uber-costa-rica-envia-carta-abierta-al-gobierno/

Fernández, H. (s.f.). Economía digital: claves y retos del internet. https://economiatic.com/economia-digital/

Fuster, M. (28 de enero del 2019). Retos y oportunidades de la irrupción de la economía de plataformas. El Pais. https://retina.elpais.com/retina/2019/01/25/innovacion/1548419632_629939.html 
Revista de Política Económica y Desarrollo Sostenible • EISSN: 2215-4167

Vol. 7 (1) • Julio-Diciembre, 2021: 1-19

Espinoza González y

DOI: https://doi.org/10.15359/peds.7-1.2

Matarrita Lorente

URL: http://www.revistas.una.ac.cr/politicaeconomica

Hidalgo, K. (22 de setiembre del 2020). DiDi y Uber piden regular su situación pero evitan decir qué impacto tendrá en sus tarifas. Amelia Rueda. https://www.ameliarueda.com/nota/uber-didiregulacion-servicio-movilidad-plataformas-noticias-costa-rica

Katz, R. y Callorda, F. (2015). Impacto de arreglos institucionales en la digitalización y el desarrollo económico de América Latina. Proceedings of the 9th CPR LATAM Conference, Cancún, 14 y 15 de julio, 2015. http://www.teleadvs.com/wp-content/uploads/Katz-Callorda-2015version-final.pdf

Lizano, A. (2018). Diseño de un prototipo de aplicación móvil para personas que han desertado de la educación secundaria en Costa Rica. San José: UNED. https://repositorio.uned.ac.cr/bitstream/ handle/120809/1787/TFG_Alvaro_Lizano_FINAL_2018_scrd.pdf?sequence=1\&isAllowe$\mathrm{d}=\mathrm{y} \#$ page $=83 \&$ zoom $=100,93,538$

Madrigal, R. (25 de agosto del 2015). ¿Uber es una "comunidad de autoabastecimiento”?. CR Hoy.com. https://archivo.crhoy.com/uber-es-una-comunidad-de-autoabastecimiento/nacionales/

Madriz, A. (05 de agosto del 2020). Uber: Urge discutir el proyecto que regula las plataformas tecnológicas de transporte. El Periódico CR. https://elperiodicocr.com/uber-urge-discutir-elproyecto-que-regula-las-plataformas-tecnologicas-de-transporte/

Ministerio Federal de Trabajo de Alemania (2017). Digitalización y el futuro del trabajo. Sinopsis del estudio “Trabajar 4.0” Análisis № 20 - 2017. https://library.fes.de/pdf-files/bueros/ argentinien/13617.pdf

Morales, J. (12 de enero del 2020). Así se ha regulado Uber en otros países. El Tiempo. https://www. eltiempo.com/tecnosfera/apps/asi-se-ha-regulado-uber-en-otros-paises-del-mundo-450998

Liang, T. P., Lin, Y. L. \& Hou, H. C. (2021). What drives consumers to adopt a sharing platform: An integrated model of value-based and transaction cost theories. Information $\sigma^{\circ}$ Management, 58(4), 103471.

París, M. (20 de marzo del 2020). Servicio público y plataformas digitales de transporte. La República. https://www.larepublica.net/noticia/servicio-publico-y-plataformas-digitales-de-transporte

Pérez, C. (2010). Revoluciones tecnológicas y paradigmas tecno-económicos. Cambridge Journal of Economics, 34(1), 185-202.

Vargas, D. (2019). Taxistas contra texto sobre Uber planteado por diputados: "Ya no vamos a aceptar ninguna regulación". Amelia Rueda. https://www.ameliarueda.com/nota/taxistas-texto-uberplanteado-diputados-no-vamos-aceptar-regulaciones 
Revista de Política Económica y Desarrollo Sostenible • EISSN: 2215-4167

Vol. 7 (1) • Julio-Diciembre, 2021: 1-19

Espinoza González y

DOI: https://doi.org/10.15359/peds.7-1.2

Matarrita Lorente

URL: http://www.revistas.una.ac.cr/politicaeconomica

Anexo 1. Resumen de las propuestas del proyecto de la Ley Reguladora de los Servicios de Transporte de Personas por Medio de Plataformas Tecnológicas (21.587)

\begin{tabular}{|c|c|c|c|}
\hline Cliente & Propietario del vehículo & Empresa o plataforma digital & Taxista \\
\hline $\begin{array}{l}\text { Pagar el } 13 \% \text { del IVA } \\
\text { sobre el precio de } \\
\text { cada viaje. }\end{array}$ & $\begin{array}{l}\text { Cumplir con requisitos } \\
\text { mínimos del vehículo } \\
\text { como: título de propiedad, } \\
\text { derecho de circulación } \\
\text { vigente, póliza de seguro, } \\
\text { el modelo para prestar el } \\
\text { servicio no podrá ser mayor } \\
\text { a ocho años de fabricación, } \\
\text { y capacidad máxima de } \\
\text { siete pasajeros. }\end{array}$ & $\begin{array}{l}\text { Inscribirse en el registro digital } \\
\text { de Empresas de Servicios de } \\
\text { Transporte de Personas (ESTP) } \\
\text { por medio de plataforma } \\
\text { tecnológica que estará a cargo del } \\
\text { Ministerio de Obras Públicas y } \\
\text { Transportes (MOPT). }\end{array}$ & $\begin{array}{l}\text { Taxis con modelos menores } \\
\text { a ocho años solo tendrán que } \\
\text { hacer una revisión técnica al } \\
\text { año (en lugar de dos), mientras } \\
\text { que modelos con más de ocho } \\
\text { años irán una vez cada seis } \\
\text { meses. }\end{array}$ \\
\hline \multirow[t]{4}{*}{$\begin{array}{l}\text { Suscribir un acuerdo } \\
\text { previo de prestación } \\
\text { del servicio con } \\
\text { los conductores de } \\
\text { plataformas digitales } \\
\text { por medio de la app. }\end{array}$} & $\begin{array}{l}\text { Inscribirse como trabajador } \\
\text { independiente ante la Caja } \\
\text { Costarricense de Seguro } \\
\text { Social (CCSS). }\end{array}$ & $\begin{array}{l}\text { Pagar } 20 \text { salarios base }(\$ 8,9 \\
\text { millones) para hacer efectiva la } \\
\text { inscripción. Ese dinero entrará } \\
\text { a la Tesorería Nacional, y el } \\
\text { registro tendrá una vigencia de } \\
\text { cuatro años. }\end{array}$ & $\begin{array}{l}\text { Se autoriza la colocación de } \\
\text { publicidad, dentro y fuera del } \\
\text { taxi. }\end{array}$ \\
\hline & $\begin{array}{l}\text { Inscribirse como } \\
\text { contribuyente ante el } \\
\text { Ministerio de Hacienda en } \\
\text { la modalidad de conductor } \\
\text { de transporte de personas } \\
\text { por medio de plataformas } \\
\text { digitales. }\end{array}$ & $\begin{array}{l}\text { Creary mantener actualizada una } \\
\text { base de datos de los conductores } \\
\text { y vehículos registrados en su } \\
\text { plataforma tecnológica. }\end{array}$ & $\begin{array}{l}\text { Podrían negociar con sus } \\
\text { clientes una nueva tarifa } \\
\text { por debajo de la fijada por } \\
\text { la ARESEP en las "marías" } \\
\text { (taxímetros). }\end{array}$ \\
\hline & $\begin{array}{l}\text { Pagar cada año un } 20 \% \\
\text { del salario base }(\$ 89.240) \\
\text { para obtener el derecho de } \\
\text { operación. }\end{array}$ & $\begin{array}{l}\text { Emitir un comprobante de pago } \\
\text { a los clientes por cada servicio } \\
\text { contratado con los siguientes } \\
\text { datos: precio, ruta transitada, } \\
\text { placa, tipo de vehículo, nombre } \\
\text { completo y foto del conductor } \\
\text { acreditado. }\end{array}$ & \\
\hline & $\begin{array}{l}\text { Suscribir un acuerdo } \\
\text { previo de prestación del } \\
\text { servicio con los clientes por } \\
\text { medio de la app. }\end{array}$ & $\begin{array}{l}\text { Remitir al MOPT, de manera } \\
\text { trimestral, el listado de los } \\
\text { conductores acreditados y } \\
\text { registrados en la plataforma } \\
\text { tecnológica. }\end{array}$ & \\
\hline
\end{tabular}

Fuente: Elfinanciero.com, 2019. 